\title{
Sound in a system of chiral one-dimensional fermions
}

\author{
K. A. Matveev \\ Materials Science Division, Argonne National Laboratory, Argonne, Illinois 60439, USA
}

(Dated: September 28, 2020)

\begin{abstract}
We consider a system of one-dimensional fermions moving in one direction, such as electrons at the edge of a quantum Hall system. At sufficiently long time scales the system is brought to equilibrium by weak interactions between the particles, which conserve their total number, energy, and momentum. Time evolution of the system near equilibrium is described by hydrodynamics based on the three conservation laws. We find that the system supports three sound modes. In the low temperature limit one mode is a pure oscillation of particle density, analogous to the ordinary sound. The other two modes involve oscillations of both particle and entropy densities. In the presence of disorder, the first sound mode is strongly damped at frequencies below the momentum relaxation rate, whereas the other two modes remain weakly damped.
\end{abstract}

Small perturbations of density propagate through ordinary fluids in the form of sound waves [1]. Oscillations of density in a sound wave are accompanied by oscillations of entropy density, such that the entropy per particle retains its equilibrium value. An important exception to the above picture is superfluid ${ }^{4} \mathrm{He}$, whose quantum nature results in the existence of two sound modes. The first sound is predominantly a wave of density, similar to sound in ordinary fluids, whereas the second sound is a wave of entropy $[1,2]$.

It was recently shown that the superfluid behavior characterized by the existence of two sound modes is a generic feature of the one-dimensional quantum fluids $[3,4]$. This is a consequence of the fact that in addition to the number of particles, energy, and momentum, the collisions of particles in these systems conserve an additional quantity $J$. The latter has the meaning of the difference of the numbers of the right- and left-moving particles. Although the scattering processes changing $J$ are not strictly forbidden, their rate is exponentially small at low temperatures, $\tau^{-1} \propto e^{-D / T}[5-7]$. (Here $D$ is the bandwidth of the system and $T$ is the temperature.) At low frequencies $\omega \ll \tau^{-1}$ the conservation of $J$ is violated, and the system behaves as an ordinary fluid, with a single sound mode. On the other hand, in a broad range of frequencies between $\tau^{-1}$ and the much larger rate of quasiparticle relaxation $\tau_{\mathrm{ex}}^{-1}$, the additional conservation law changes the dynamics of the quantum fluid dramatically, resulting in two sound modes.

In this paper we study sound in a gas of chiral onedimensional fermions. The best known example of such a system is the edge of the two-dimensional electron gas in the integer quantum Hall regime [8]. The chiral nature of transport in this system was demonstrated by observing propagation of the density pulses along the edge [9] More recently, an analog of the quantum Hall system was created with cold atoms, and the chiral nature of the edge states has also been demonstrated experimentally [10]. We show below that the number of sound modes in a chiral system is equal to the number of conserved quantities. In the absence of disorder, collisions between fermions conserve the number of particles, energy, and momentum, resulting in three sound modes. By breaking the conservation of momentum, static disorder reduces the number of sound modes to two. This is in contrast to nonchiral systems, where no sound may propagate in the presence of disorder.

We focus on the simplest case of a single-component chiral Fermi gas, which corresponds to occupation fraction $\nu=1$ in the quantum Hall realizations of the system. At low temperatures, the energy spectrum of fermions, which we assume to be spinless, can be expanded in momentum near the Fermi point,

$$
\epsilon_{p}=v_{F} p+\frac{p^{2}}{2 m}+\frac{\lambda p^{3}}{2 m^{2} v_{F}}+\ldots
$$

Here we measure the momentum $p$ from the Fermi point, $v_{F}$ is the Fermi velocity, parameter $m$ has the dimension of mass, while $\lambda$ is dimensionless. For simplicity, we assume that the interactions between particles are weak and account for them only to the extent that they bring the system to thermal equilibrium, with the characteristic relaxation rate $\tau_{\mathrm{ex}}^{-1}$. Stronger interactions would alter our results for the sound velocities but not the fundamental features, such as the number of the sound modes.

We note that it is important to properly account for the nonlinear corrections to the energy spectrum in Eq. (1). Indeed, for the linear spectrum the energy of the system is determined by its momentum, $E=v_{F} P$, and the two corresponding conservation laws become equivalent. Because of that, the subsequent calculations simplify considerably if instead of conservation of momentum one discusses conservation of the quantity $\Theta=E-v_{F} P$. In this approach, the equilibrium state of the system is fully determined by specifying the total number of particles $N$, energy $E$, and $\Theta$. Correspondingly, the occupation numbers of the fermionic states

$$
f_{p}=\frac{1}{e^{\left(\epsilon_{p}-\mu-\gamma \theta_{p}\right) / T}+1}, \quad \theta_{p}=\epsilon_{p}-v_{F} p,
$$

are controlled by three parameters: the chemical potential $\mu$, the temperature $T$, and the dimensionless param- 
eter $\gamma$. The values of $\mu, T$, and $\gamma$ are determined by the magnitudes of the conserved quantities $N, E$, and $\Theta$.

We are interested in the dynamics of the system at frequencies $\omega$ well below the quasiparticle relaxation rate $\tau_{\text {ex }}^{-1}$. In this regime, the Fermi-Dirac form (2) of the occupation numbers applies at every point in space, but the parameters $\mu, T$, and $\gamma$ may depend on the position $x$ and time $t$. The dynamics of the system is fully described by the continuity equations expressing the three conservation laws,

$$
\begin{gathered}
\partial_{t} n+\partial_{x} j_{n}=0, \\
\partial_{t} \varepsilon+\partial_{x} j_{\varepsilon}=0, \\
\partial_{t} \vartheta+\partial_{x} j_{\vartheta}=0 .
\end{gathered}
$$

Here $n, \varepsilon$, and $\vartheta$ are the densities of $N, E$, and $\Theta$, respectively, whereas $j_{n}, j_{\varepsilon}$, and $j_{\vartheta}$ are the corresponding currents. For noninteracting fermions they are given by

$$
\begin{aligned}
& n=\int \frac{d p}{h} f_{p}, \quad \varepsilon=\int \frac{d p}{h} \epsilon_{p} f_{p}, \quad \vartheta=\int \frac{d p}{h} \theta_{p} f_{p}, \\
& j_{n}=\int \frac{d p}{h} v_{p} f_{p}, \quad j_{\varepsilon}=\int \frac{d p}{h} v_{p} \epsilon_{p} f_{p}, \quad j_{\vartheta}=\int \frac{d p}{h} v_{p} \theta_{p} f_{p} .
\end{aligned}
$$

where $h$ is the Planck's constant and $v_{p}=\partial_{p} \epsilon_{p}$ is the velocity of the fermion with momentum $p$. Substitution of the densities and currents defined by Eq. (4) with $f_{p}$ given by Eq. (2) into the continuity equations (3) gives three equations upon the three parameters $\mu(x, t), T(x, t)$, and $\gamma(x, t)$ of the distribution function.

To obtain sound modes of the system, we assume that these parameters oscillate according to

$$
\begin{aligned}
& \mu(x, t)=\delta \mu e^{i(q x-\omega t)}, \quad T(x, t)=T+\delta T e^{i(q x-\omega t)}, \\
& \gamma(x, t)=\delta \gamma e^{i(q x-\omega t)},
\end{aligned}
$$

with small amplitudes $\delta \mu, \delta T$, and $\delta \gamma$ and linearize Eq. (3) in these parameters. This yields a system of three linear equations, which we write in the matrix form

$$
(\omega \widehat{D}-q \widehat{J}) \Psi=0
$$

Here

$$
\widehat{D}=\left(\begin{array}{lll}
\partial_{\mu} n & \partial_{T} n & \partial_{\gamma} n \\
\partial_{\mu} \varepsilon & \partial_{T} \varepsilon & \partial_{\gamma} \varepsilon \\
\partial_{\mu} \vartheta & \partial_{T} \vartheta & \partial_{\gamma} \vartheta
\end{array}\right), \quad \widehat{J}=\left(\begin{array}{lll}
\partial_{\mu} j_{n} & \partial_{T} j_{n} & \partial_{\gamma} j_{n} \\
\partial_{\mu} j_{\varepsilon} & \partial_{T} j_{\varepsilon} & \partial_{\gamma} j_{\varepsilon} \\
\partial_{\mu} j_{\vartheta} & \partial_{T} j_{\vartheta} & \partial_{\gamma} j_{\vartheta}
\end{array}\right)
$$

and the column vector $\Psi=(\delta \mu, \delta T, \delta \gamma)^{\mathrm{T}}$. The sound modes are given by nonvanishing solutions of Eq. (6), which exist only when $\omega$ and $q$ satisfy the condition

$$
\operatorname{det}(\omega \widehat{D}-q \widehat{J})=0
$$

For a given $q$, the above condition is a cubic equation for $\omega$, which in general has three solutions. This conclusion applies to any chiral system with conserved number of particles, energy, and momentum.

In the particular case of the gas of chiral fermions at low temperature, further progress can be made by evaluating the matrices (7) to leading order in $T / m v_{F}^{2}$ using Eqs. (4) and (2). We obtain

$$
\widehat{D}=\left(\begin{array}{ccc}
\frac{1}{h v_{F}} & -\frac{\pi^{2} T}{3 h m v_{F}^{3}} & \frac{\pi^{2} T^{2}}{6 h m v_{F}^{3}} \\
-\frac{\pi^{2} T^{2}}{3 h m v_{F}^{3}} & \frac{\pi^{2} T}{3 h v_{F}} & \frac{7 \pi^{4}(\lambda-2) T^{4}}{30 h m^{2} v_{F}^{5}} \\
\frac{\pi^{2} T^{2}}{6 h m v_{F}^{3}} & \frac{7 \pi^{4}(\lambda-2) T^{3}}{30 h m^{2} v_{F}^{5}} & \frac{7 \pi^{4} T^{4}}{60 h m^{2} v_{F}^{5}}
\end{array}\right)
$$

and

$$
\widehat{J}=\left(\begin{array}{ccc}
\frac{1}{h} & 0 & \frac{\pi^{2} T^{2}}{6 h m v_{F}^{2}} \\
0 & \frac{\pi^{2} T}{3 h} & \frac{7 \pi^{4}(\lambda-1) T^{4}}{30 h m^{2} v_{F}^{4}} \\
\frac{\pi^{2} T^{2}}{6 h m v_{F}^{2}} & \frac{7 \pi^{4}(\lambda-1) T^{3}}{30 h m^{2} v_{F}^{4}} & \frac{7 \pi^{4} T^{4}}{60 h m^{2} v_{F}^{4}}
\end{array}\right) .
$$

Then by solving Eq. (8) we find three values of the sound velocity $\omega / q$,

$$
v_{1}=v_{F}, \quad v_{ \pm}=v_{F}\left(1 \pm \sqrt{\frac{7}{5}} \frac{\pi T}{m v_{F}^{2}}\right) .
$$

where we kept only the terms up to first order in $T / m v_{F}^{2}$. In this approximation the relative magnitudes of the oscillations of $\mu, T$, and $\gamma$ are given by

$$
\delta T=0, \quad \frac{\delta \mu}{\delta \gamma}=-\frac{7}{10} \frac{\pi^{2} T^{2}}{m v_{F}^{2}},
$$

for the mode propagating at velocity $v_{1}=v_{F}$ and

$$
\delta \mu=0, \quad \frac{\delta T}{\delta \gamma}= \pm \sqrt{\frac{7}{20}} \frac{\pi T^{2}}{m v_{F}^{2}},
$$

for the solutions with sound velocities $v_{ \pm}$. One can use the expression (9) for the matrix $\widehat{D}$ to obtain the relative magnitude of the oscillations of density $\delta n$ and entropy density $\delta s=\delta \varepsilon / T$. At $T / m v_{F}^{2} \rightarrow 0$ one finds $\delta s / \delta n=$ 0 for the solution with velocity $v_{1}=v_{F}$ and $\delta s / \delta n=$ $\pm \pi \sqrt{7 / 5}$ for the modes with velocities $v_{ \pm}$.

Let us now discuss the effect of static disorder on the sound modes. Disorder breaks the translation invariance of the system, and the total momentum is no longer a conserved quantity. This brings about two changes in the above discussion. First, the parameter $\gamma$ in the FermiDirac distribution (2) of the occupation numbers is now permanently set to zero. Second, only the continuity equations (3a) and (3b) are satisfied. Upon linearization 
in $\delta \mu$ and $\delta T$, Eq. (6) still applies, but the matrices $\widehat{D}$ and $\widehat{J}$ are given by the first two rows and columns of the corresponding expressions in Eq. (7), and $\Psi=(\delta \mu, \delta T)^{\mathrm{T}}$. At a given $q$, the condition (8) is now a quadratic equation for $\omega$. It has two solutions corresponding to two sound modes. For the chiral Fermi gas at low temperatures we solve Eq. (8) using the matrices $\widehat{D}$ and $\widehat{J}$ given by the first two rows and columns of Eqs. (9) and (10). The resulting sound velocity $\omega / q$ takes two possible values

$$
v_{ \pm}=v_{F}\left(1 \pm \frac{\pi}{\sqrt{3}} \frac{T}{m v_{F}^{2}}\right) .
$$

Here again we omitted the terms of second and higher powers in $T$. The eigenvectors $\Psi$ corresponding to these two solutions have $\delta \mu / \delta T= \pm \pi / \sqrt{3}$. The corresponding oscillations of density $\delta n$ and entropy density $\delta s$ in the two sound modes satisfy the conditions $\delta s / \delta n= \pm \pi / \sqrt{3}$.

To gain further insight into the effect of disorder on the sound modes, we consider the case of weak disorder. The latter condition means that the rate of relaxation of momentum $\tau_{P}^{-1}$ due to disorder is small compared to the quasiparticle relaxation rate $\tau_{\mathrm{ex}}^{-1}$. At $\omega \ll \tau_{\mathrm{ex}}^{-1}$ the quasiparticles of the Fermi gas are in equilibrium, and the occupation numbers take the form (2), but the parameter $\gamma$ experiences relaxation due to the momentum nonconserving scattering processes. Assuming this relaxation takes the usual form

$$
\dot{\gamma}=-\frac{\gamma}{\tau_{P}}
$$

we now obtain the effect of these processes on the three sound modes.

First, we note that a nonzero $\dot{\gamma}$ is accompanied by nonzero $\dot{\mu}$ and $\dot{T}$. Indeed, the remaining laws of conservation of the particle number and energy imply $\dot{n}=0$ and $\dot{\varepsilon}=0$. The set of three quantities $\dot{\Phi}=(\dot{n}, \dot{\varepsilon}, \dot{\vartheta})^{\mathrm{T}}$ is obtained from $\dot{\Psi}=(\dot{\mu}, \dot{T}, \dot{\gamma})^{\mathrm{T}}$ with the help of the matrix $\widehat{D}$ [see Eq. (7)], i.e., $\dot{\Phi}=\widehat{D} \dot{\Psi}$. We then obtain $\dot{\mu}$ and $\dot{T}$ by imposing the condition that the first two components of $D \dot{\Psi}$ vanish. The remaining component $\dot{\vartheta}$ then takes the value

$$
\dot{\vartheta}=\frac{4 \pi^{4} T^{4}}{45 h m^{2} v_{F}^{5}} \dot{\gamma},
$$

where we used Eq. (9) for $\widehat{D}$. It has the meaning of the rate of change of the density of $\Theta$ due to the scattering processes violating conservation of momentum.

Next, we add $\dot{\vartheta}$ given by Eq. (16) with $\dot{\gamma}$ given by Eq. (15) to the right-hand side of Eq. (3c) and solve the system of equations (3) in linear order in small $\delta \mu, \delta T$, and $\delta \vartheta$ introduced via Eq. (5). In the low-temperature regime $T \ll m v_{F}^{2}$, the frequency $\omega$ for a given $q$ can be presented as

$$
\omega=v_{F} q\left(1+\frac{T}{m v_{F}^{2}} x\right)
$$

where $x$ satisfies

$$
x^{3}+\frac{i x^{2}}{\eta}-\frac{7 \pi^{2} x}{5}-\frac{i \pi^{2}}{3 \eta}=0, \quad \eta=\frac{q T}{m v_{F}} \tau_{P} .
$$

This cubic equation has three solutions $x(\eta)$ that correspond to the three sound modes. Although analytic expressions for these solutions can be obtained, their form is overly complicated. Thus, we focus on the important limiting cases of $\eta \rightarrow 0$ and $\eta \rightarrow \infty$.

One of the three solutions is purely imaginary. In the limiting cases we find $x=-i / \eta$ at $\eta \rightarrow 0$ and $x=-(5 / 21) i / \eta$ at $\eta \rightarrow \infty$. This solution corresponds to the sound mode that propagates at velocity $v_{1}=v_{F}$ at high $q$. The other two solutions of Eq. (18) yield the modes propagating with velocities $v_{ \pm}$. For these solutions we find $x= \pm \pi / \sqrt{3}-8 \pi^{2} i \eta / 15$ at $\eta \rightarrow 0$ and $x= \pm \sqrt{7 / 5} \pi-(8 / 21) i / \eta$ at $\eta \rightarrow \infty$. Using these expressions, we obtain the frequencies of the sound modes in the limits of small and large $q$. At $q \gg m v_{F} / T \tau_{P}$ our results for $x$ at $\eta \rightarrow \infty$ upon substitution into Eq. (17) yield

$$
\omega=v_{F} q-\frac{5 i}{21 \tau_{P}}, \quad \omega=v_{F}\left(1 \pm \sqrt{\frac{7}{5}} \frac{\pi T}{m v_{F}^{2}}\right) q-\frac{8 i}{21 \tau_{P}} .
$$

At $\tau_{P} \rightarrow \infty$ Eq. (19) recovers our earlier results for the three sound modes in the absence of momentum relaxation with the sound velocities given by Eq. (11). At finite $\tau_{P}$ the frequencies of the sound modes acquire imaginary parts of the order of $-\tau_{P}^{-1}$. The latter are the rates of attenuation of sound due to the momentum relaxation processes.

Our expressions for $x$ at $\eta \rightarrow 0$ yield the sound mode frequencies at $q \ll m v_{F} / T \tau_{P}$,

$\omega=v_{F} q-\frac{i}{\tau_{P}}, \quad \omega=v_{F} q\left(1 \pm \frac{\pi T}{\sqrt{3} m v_{F}^{2}}\right)-\frac{8 \pi^{2} i q^{2} T^{2} \tau_{P}}{15 m^{2} v_{F}^{2}}$.

At $q \rightarrow 0$ the first mode is overdamped, while the other two experience only weak attenuation. Their velocities recover our earlier result (14).

The effect of weak static disorder on the three sound modes can be summarized as follows. At high $q$ each of the three modes is attenuated with the rate of the order of $\tau_{P}^{-1}$. However, their behavior at lower $q$ is dramatically different. The sound mode propagating with velocity $v_{1}=v_{F}$ remains attenuated with the rate of the order of $\tau_{P}^{-1}$, and at $\omega \lesssim \tau_{P}^{-1}$ this sound mode ceases to exist. On the other hand, the attenuation rates of the other two modes depend on $q$ in such a way that they remain weak (i.e., small compared to the real part of $\omega$ ) at all frequencies. The main effect of the momentum relaxation is the crossover of the sound velocities $v_{ \pm}$from their values given by Eq. (11) at $\omega \tau_{P} \gg m v_{F}^{2} / T$ to Eq. (14) at $\omega \tau_{P} \ll m v_{F}^{2} / T$. 
It is instructive to compare the behavior of sound modes in chiral one-dimensional systems with that in nonchiral ones. In the latter case the nature of the two sound modes depends on the presence of spins in the system. For fermions with spin, the two modes are very similar to sound modes in superfluid ${ }^{4} \mathrm{He}[1,2]$. In particular, they have different velocities at $T \rightarrow 0$, the first sound is a wave of particle density, whereas the second sound is the wave of entropy [3]. For spinless one-dimensional quantum fluids the two sound modes have the same velocity at $T \rightarrow 0$. At small but finite temperature the degeneracy is split, but the two resulting modes are hybrids of the first and second sounds: the amplitudes of the oscillations of density $\delta n$ and entropy density $\delta s$ are of the same order of magnitude [4]. The two sound modes in the case of chiral fermions with disorder are of a similar hybrid nature, with both velocities approaching $v_{F}$ at $T \rightarrow 0$ [see Eq. (14)] and $\delta s / \delta n= \pm \pi / \sqrt{3}$. In the absence of disorder, the modes propagating with velocities $v_{ \pm}$are also hybrids, with $\delta s / \delta n= \pm \pi \sqrt{7 / 5}$, whereas the additional mode propagating at velocity $v_{F}$ is a wave of density, analogous to the first sound: $\delta s / \delta n \rightarrow 0$ at $T \rightarrow 0$. Similarly to the ordinary sound, this mode is effectively damped at small $q$ in the presence of disorder. On the other hand, disorder results in only weak attenuation of the two hybrid modes.

It is important to distinguish between the sound modes discussed in this paper and the bosonic excitations in the Luttinger liquid theory [11]. Indeed, our model of chiral spinless fermions can be bosonized, resulting in an alternative theory based on bosonic rather than fermionic elementary excitations. The bosons are essentially quantized waves of particle density that propagate at the Fermi velocity. The nonlinear corrections to the energy spectrum (1) translate into anharmonic coupling of bosons [11], resulting in a finite lifetime $\tau_{b} \sim \hbar m v_{F}^{2} / T^{2}$ for excitations with energy of order $T$ [12]. The lifetime $\tau_{b}$ is usually shorter than the lifetime of fermionic quasiparticles $\tau_{\mathrm{ex}}[13,14]$. At time scales beyond $\tau_{\mathrm{ex}}$, neither type of quasiparticles remains well defined, and the hydrodynamic description of the fluid presented here becomes appropriate.

The two sound modes in the disordered system can be studied in an experiment with quantum Hall devices, where propagation of a density pulse along the edge is observed. At time scales $t \gg \tau_{\text {ex }}$ the pulse is expected to split in two, propagating with velocities $v_{+}$and $v_{-}$ given by Eq. (14). An experiment of this type was reported in Ref. [9], where the density pulse was created by a local gate. The propagation of the density pulse was observed, but it did not split into two pulses. A possible reason is that at the very low measurement temperature of $0.3 \mathrm{~K}$ the relaxation time $\tau_{\text {ex }}$ may have exceeded the time during which the pulse was observed. For spinless chiral fermions $\tau_{\text {ex }}$ scales with the temperature as $T^{-2}$ and $T^{-6}[13]$ for pure Coulomb interactions and those screened by a nearby gate, respectively, and as $T^{-14}$ in the case of very short range interactions [14]. Thus the conditions for the observation of sound modes are more favorable at higher temperatures. We mention finally that in the case of a disorder-free system, only the first sound mode is efficiently coupled to the gate, because to leading order the oscillations of the chemical potential in the hybrid modes vanish, see Eq. (13).

To summarize, we have studied sound modes in a system of chiral one-dimensional weakly interacting fermions. In the absence of disorder the system supports three sound modes: the first sound propagating at the Fermi velocity and two hybrid modes with velocities $v_{ \pm}$ different from $v_{F}$ by $\delta v \sim \pm T / m v_{F}$. Disorder effectively damps the first sound, but the hybrid modes propagate with little damping.

The author is grateful to A. V. Andreev for helpful discussions. This work was supported by the U.S. Department of Energy, Office of Science, Basic Energy Sciences, Materials Sciences and Engineering Division.

[1] L. D. Landau and E. M. Lifshitz, Fluid Mechanics (Elsevier, Oxford, 2013).

[2] I. M. Khalatnikov, An introduction to the theory of superfluidity (Perseus, New York, 2000).

[3] K. A. Matveev and A. V. Andreev, Second Sound in Systems of One-Dimensional Fermions, Phys. Rev. Lett. 119, 266801 (2017).

[4] K. A. Matveev and A. V. Andreev, Hybrid Sound Modes in One-Dimensional Quantum Liquids, Phys. Rev. Lett. 121, 026803 (2018).

[5] A. M. Lunde, K. Flensberg, and L. I. Glazman, Threeparticle collisions in quantum wires: Corrections to thermopower and conductance, Phys. Rev. B 75, 245418 (2007).

[6] T. Micklitz, J. Rech, and K. A. Matveev, Transport properties of partially equilibrated quantum wires, Phys. Rev. B 81, 115313 (2010).

[7] K. A. Matveev and A. V. Andreev, Equilibration of a spinless Luttinger liquid, Phys. Rev. B 85, 041102(R) (2012).

[8] B. I. Halperin, Quantized Hall conductance, currentcarrying edge states, and the existence of extended states in a two-dimensional disordered potential, Phys. Rev. B 25, 2185 (1982).

[9] R. C. Ashoori, H. L. Stormer, L. N. Pfeiffer, K. W. Baldwin, and K. West, Edge magnetoplasmons in the time domain, Phys. Rev. B 45, 3894 (1992).

[10] N. Goldman, J. Dalibard, A. Dauphin, F. Gerbier, M. Lewenstein, P. Zoller, and I. B. Spielman, Direct imaging of topological edge states in cold-atom systems, PNAS 110, 6736 (2013).

[11] F. D. M. Haldane, 'Luttinger liquid theory' of onedimensional quantum fluids. I. Properties of the Luttinger model and their extension to the general 1D interacting spinless Fermi gas, J. Phys. C: Solid State Phys. 14, 2585 (1981).

[12] K. V. Samokhin, Lifetime of excitations in a clean Lut- 
tinger liquid, J. Phys. Condens. Matter 10, L533 (1998).

[13] Z. Ristivojevic and K. A. Matveev, Relaxation of weakly interacting electrons in one dimension, Phys. Rev. B 87, 165108 (2013).
[14] I. V. Protopopov, D. B. Gutman, and A. D. Mirlin, Equilibration in a chiral Luttinger liquid, Phys. Rev. B 91, 195110 (2015). 